\title{
Globally Asymptotic Stability of Stochastic Nonlinear Systems with Time-Varying Delays via Output Feedback Control
}

\author{
Mingzhu Song, ${ }^{1}$ Wenwen Cheng, ${ }^{2}$ Quanxin Zhu, ${ }^{2,3}$ Hongwei Zhou, ${ }^{4}$ and Hui Wang ${ }^{2}$ \\ ${ }^{1}$ Department of Mathematics and Computer Science, Tongling University, Tongling 244000, China \\ ${ }^{2}$ School of Mathematical Sciences and Institute of Finance and Statistics, Nanjing Normal University, Nanjing 210023, China \\ ${ }^{3}$ Department of Mathematics, University of Bielefeld, 33615 Bielefeld, Germany \\ ${ }^{4}$ School of Mathematics and Information Technology, Nanjing Xiaozhuang University, Nanjing, Jiangsu 211171, China
}

Correspondence should be addressed to Hongwei Zhou; davidchou@njxzc.edu.cn

Received 24 October 2015; Accepted 17 February 2016

Academic Editor: Cengiz Çinar

Copyright (c) 2016 Mingzhu Song et al. This is an open access article distributed under the Creative Commons Attribution License, which permits unrestricted use, distribution, and reproduction in any medium, provided the original work is properly cited.

We address the problem of globally asymptotic stability for a class of stochastic nonlinear systems with time-varying delays. By the backstepping method and Lyapunov theory, we design a linear output feedback controller recursively based on the observable linearization for a class of stochastic nonlinear systems with time-varying delays to guarantee that the closed-loop system is globally asymptotically stable in probability. In particular, we extend the deterministic nonlinear system to stochastic nonlinear systems with time-varying delays. Finally, an example and its simulations are given to illustrate the theoretical results.

\section{Introduction}

In recent years, the stochastic nonlinear system has received much attention and has enjoyed a good development, which has been widely applied in many fields such as engineering and finance [1]. Particularly, owing to the reason that a system by the output feedback is more flexible to respond to the information of control systems, the stochastic nonlinear system via the output feedback control has been more widely studied. Marino and Tomei [2] and Battilotti [3] designed linear observer to study the output feedback control for nonlinear systems. As a result, a large number of researchers have been focused on the stability analysis of stochastic nonlinear systems and the design of controller.

Since the backstepping method has been introduced in the nonlinear system, the theory of stochastic nonlinear systems has achieved a remarkable development. According to the different Lyapunov function, there are two ways to solve the problem. One is that Pan and Basar [4-6] considered a quadratic Lyapunov function. By using the backstepping method to design the controller, they discussed the risksensitive optimal control problem. The other is that Deng and
Krstić [7-10] used a quartic Lyapunov function to guarantee that the closed-loop system is globally asymptotically stable in probability. Based on their works, Liu et al. [11-13] employed the quartic Lyapunov function to design the output feedback control for stochastic nonlinear systems. M.-L. Liu and Y.-G. Liu [14] and Chen et al. [15] used such Lyapunov function to study the state feedback stability for stochastic nonlinear systems with time-varying delays. In [16], Du et al. discussed the global output feedback stability for a class of uncertain upper-triangular systems with the input delay. Finite-time stability for stochastic nonlinear systems in strictfeedback form was considered in [17]. More results can be found in [18-20].

In this paper, we are concerned with the globally asymptotic stability of stochastic nonlinear system with timevarying delays. We extend the results of the deterministic nonlinear systems in [21] to the stochastic nonlinear systems with time-varying delays by output feedback control and design a Lyapunov-Krasovskii functional to prove the globally asymptotic stability of the closed-system via the linear observer. It is obvious that the form of the linear controller is simpler than that used in [9]. And compared with the 
$C^{1}$ output feedback controller obtained by the homogeneous observers in [20], the controller designed by the linear observers in this paper is smooth.

The rest of this paper is organized as follows. In Section 2, we present some definitions and establish a new inequality, which plays an important role in the proof of our main results. In Section 3, we construct a Lyapunov-Krasovskii functional and design a linear output feedback control to prove the globally asymptotic stability based on the linear observers. In Section 4, we use an example to illustrate our theoretical results. Finally, we conclude this paper with some general remarks.

\section{Preliminaries}

In this section, we will give the following notations, definitions, and some preliminary lemmas. $\mathbb{R}_{+}$denotes the set of all nonnegative real numbers; $\mathbb{R}^{n}$ denotes the real $n$ dimensional space; Trace $\{X\}$ denotes the trace for square matrix $X ;|X|$ denotes the Euclidean norm of a vector $X$. $\mathscr{C}\left([-d, 0] ; \mathbb{R}^{n}\right)$ denotes the space of continuous $\mathbb{R}^{n}$-valued functions on $[-d, 0]$ endowed with the norm $\|\cdot\|$ defined by $\|f\|=\sup _{x \in[-d, 0]}|f(x)|$ for $f \in \mathscr{C}\left([-d, 0] ; \mathbb{R}^{n}\right)$; $\mathscr{C}_{\mathscr{F}_{0}}^{b}\left([-d, 0] ; \mathbb{R}^{n}\right)$ denotes the family of all $\mathscr{F}_{0}$-measurable bounded $\mathscr{C}\left([-d, 0] ; \mathbb{R}^{n}\right)$-valued random variables $\xi=\{\xi(\theta)$ : $-d \leq \theta \leq 0\} . \mathscr{C}^{i}$ denotes the set of all functions with continuous $i$ th partial derivatives; $\mathscr{C}^{2,1}\left(\mathbb{R}^{n} \times[-d, \infty] ; \mathbb{R}^{+}\right)$ denotes the family of all nonnegative functions $V(x, t)$ on $\mathbb{R}^{n} \times[-d, \infty)$ which are $\mathscr{C}^{2}$ in $x$ and $\mathscr{C}^{1}$ in $t ; \mathscr{C}^{2,1}$ denotes the family of all functions which are $\mathscr{C}^{2}$ in the first argument and $\mathscr{C}^{1}$ in the second argument. $\mathscr{K}$ denotes the set of all functions: $\mathbb{R}_{+} \rightarrow \mathbb{R}_{+}$, which are continuous and strictly increasing and vanish at zero; $\mathscr{K}_{\infty}$ denotes the set of all functions which are of class $\mathscr{K}$ and unbounded. $\mathscr{K} \mathscr{L}$ is the set of all functions $\beta(s, t): \mathbb{R}_{+} \times \mathbb{R}_{+} \rightarrow \mathbb{R}_{+}$, which are of $\mathscr{K}$ for each fixed $t$ and decrease to zero as $t \rightarrow \infty$ for each fixed $s$.

Consider the following stochastic time-varying delay system:

$$
\begin{aligned}
d x(t)= & f(x(t), x(t-d(t), t)) d t \\
& +g(x(t), x(t-d(t)), t) d \omega(t), \quad \forall t \geq 0,
\end{aligned}
$$

with initial date $\{x(\theta):-d \leq \theta \leq 0\}=\xi \in \mathscr{C}_{\mathscr{F}_{0}}^{b}\left([-d, 0] ; \mathbb{R}^{n}\right)$, where $d(t): \mathbb{R}_{+} \rightarrow[0, d]$ is a Borel measurable function and $\omega(t)$ is an $r$-dimensional standard Wiener process defined on the complete probability space $\left(\Omega, \mathscr{F},\left\{\mathscr{F}_{t}\right\}_{t \geq 0}, P\right)$ with $\Omega$ being a sample space, $\mathscr{F}$ being a filtration, and $P$ being a probability measure. $f: \mathbb{R}^{n} \times \mathbb{R}^{n} \times \mathbb{R}_{+} \rightarrow \mathbb{R}^{n}, g: \mathbb{R}^{n} \times \mathbb{R}^{n} \times$ $\mathbb{R}_{+} \rightarrow \mathbb{R}^{n \times r}$ are assumed to be locally Lipschitz in $(x(t), x(t-$ $d(t)))$ uniformly in $t$ and satisfy $f(0,0, t) \equiv 0, g(0,0, t) \equiv 0$.

Definition 1 (see [22]). For any given $V(x(t), t) \in \mathscr{C}^{2,1}$ associated with system (1), the differential operator $\mathscr{L}$ is defined as

$$
\mathscr{L} V(x(t), t)=\frac{\partial V}{\partial t}+\frac{\partial V}{\partial x^{T}} f+\frac{1}{2} \operatorname{Trace}\left\{g^{T} \frac{\partial^{2} V}{\partial x^{2}} g\right\},
$$

where $(1 / 2) \operatorname{Trace}\left\{g^{T}\left(\partial^{2} V / \partial x^{2}\right) g\right\}$ is called as the Hessian term of $\mathscr{L}$.

Definition 2 (see $[11,15])$. For system (1), the equilibrium $x=$ 0 is said to be globally asymptotically stable in probability, if, for any $\varepsilon>0$, there exists a class of $\mathscr{K}$ function $\gamma(\cdot)$ such that

$$
\begin{aligned}
P\{|x(t)|<\gamma(\|\xi\|)\} & \geq 1-\varepsilon, \\
\forall t & \geq 0, \forall \xi \in \mathscr{C}_{\mathscr{F}_{0}}^{b}\left([-d, 0] ; \mathbb{R}^{n}\right) \backslash\{0\},
\end{aligned}
$$

and for any $\xi \in \mathscr{C}_{\mathscr{F}_{0}}^{b}\left([-d, 0] ; \mathbb{R}^{n}\right)$, one has

$$
P\left\{\lim _{t \rightarrow \infty}|x(t)|=0\right\}=1 .
$$

Definition 3 (see [22]). For fixed coordinates $\left(x_{1}, \ldots, x_{n}\right) \in$ $\mathbb{R}^{n}$ and real numbers $r_{i}>0, i=1, \ldots, n$.

(1) The dilation $\Delta_{\epsilon}(x)$ is defined by $\Delta_{\epsilon}(x)=$ $\left(\epsilon^{r_{1}} x_{1}, \ldots, \epsilon^{r_{n}} x_{n}\right)$ for any $\epsilon>0 ; r_{1}, \ldots, r_{n}$ are called the weights of the coordinates. For simplicity, we define dilation weight $\Delta=\left(r_{1}, \ldots, r_{n}\right)$.

(2) A function $V \in \mathscr{C}\left(\mathbb{R}^{n}, R\right)$ is said to be homogeneous of degree $\tau$ if there is a real number $\tau \in R$ such that $V\left(\Delta_{\epsilon}(x)\right)=$ $\epsilon^{\tau} V\left(x_{1}, \ldots, x_{n}\right)$ for any $x \in \mathbb{R}^{n} \backslash\{0\}, \epsilon>0$.

(3) A vector field $h \in \mathscr{C}\left(\mathbb{R}^{n}, \mathbb{R}^{n}\right)$ is said to be homogeneous of degree $\tau$ if there is a real number $\tau \in R$ such that $h_{i}\left(\Delta_{\epsilon}(x)\right)=\epsilon^{\tau+r_{i}} h_{i}(x)$ for any $x \in \mathbb{R}^{n} \backslash\{0\}, \epsilon>0$.

(4) A homogeneous $q$-norm is defined as $\|x\|_{\Delta, q}=$ $\left(\sum_{i=1}^{n}\left|x_{i}\right|^{q / r_{i}}\right)^{1 / q}$ for any $x \in \mathbb{R}^{n}$, where $q>1$ is a constant. For simplicity, in this paper, we choose $q=2$ and write $\|x\|_{\Delta}$ for $\|x\|_{\Delta, 2}$.

Lemma 4 (see $[11,15,22])$. For system (1), if there exist a function $V(x(t), t) \in \mathscr{C}^{2,1}\left(R^{n} \times[-d, \infty) ; R_{+}\right)$, two class $\mathscr{K}_{\infty}$ functions $\alpha_{1}, \alpha_{2}$, and a class $\mathscr{K}$ function $\alpha_{3}$ such that

$$
\begin{aligned}
\alpha_{1}(|x(t)|) \leq V(x(t), t) \leq \alpha_{2}\left(\sup _{-d \leq \sigma \leq 0}|x(\sigma+t)|\right), \\
\mathscr{L} V(x(t), t) \leq-\alpha_{3}(|x(t)|),
\end{aligned}
$$

then there exists a unique solution on $[-d, \infty)$ for each $x_{0} \in \mathbb{R}^{n}$ and the equilibrium $x(t)=0$ is globally asymptotically stable in probability.

Lemma 5 (see [22]). Suppose that $V: \mathbb{R} \rightarrow R$ is a homogeneous function of degree $\tau$ with respect to the dilation weight $\Delta$; then

(i) $\partial V / \partial x_{i}$ is homogeneous of degree $\tau-r_{i}$ with $r_{i}$ being the homogeneous weight of $x_{i}$;

(ii) there is a constant $\bar{\gamma}$ such that $V(x(t)) \leq \bar{\gamma}\|x(t)\|_{\Delta}^{\tau}$. Moreover, if $V(x(t))$ is positive definite, then $V(x(t)) \geq$ $\gamma\|x(t)\|_{\Delta}^{\tau}$, where $\gamma$ is a positive constant.

Lemma 6 (see [1]). Let $V: D \rightarrow R$ be a continuous positive definite on a domain $D=R^{n}$ that contains the origin. Let $B_{r} C$ $D$ for some $r>0$. Then, there exist class $\mathscr{K}$ functions $\alpha_{1}$ and $\alpha_{2}$, defined on $[0, r]$, such that

$$
\alpha_{1}(|x(t)|) \leq V(x(t)) \leq \alpha_{2}(|x(t)|)
$$


for all $x \in B_{r}$. If $D=\mathbb{R}^{n}$, the functions $\alpha_{1}$ and $\alpha_{2}$ will be defined on $[0, \infty)$ and the foregoing inequality will hold for all $x \in \mathbb{R}^{n}$. Moreover, if $V(x(t))$ is radially unbounded, then $\alpha_{1}$ and $\alpha_{2}$ can be chosen to belong to class $\mathscr{K}_{\infty}$.

Lemma 7. For any constants $a>0$ and $b \in \mathbb{R}$, one has that, for any $x, y \in \mathbb{R}$,

$$
\begin{aligned}
& -a x^{4}+b x y^{3} \leq k_{1} a^{-1 / 3} b^{4 / 3} y^{4}, \\
& -a x^{4}+b x^{3} y \leq k_{2} b^{4} a^{-3} y^{4}
\end{aligned}
$$

where $k_{1}=4^{-1 / 3}-4^{-4 / 3}>0$ and $k_{2}=(3 / 4)^{3}-(3 / 4)^{4}>0$.

Proof. We first prove (8). Let $Z_{1}(x)=-a x^{4}+b x y^{3}$, where $y$ is a parameter. Then, we have

$$
Z_{1}^{\prime}(x)=-4 a x^{3}+b y^{3}=0, \quad x=\left(\frac{b}{4 a}\right)^{1 / 3} y
$$

It is clear that, for any $x \in\left(-\infty,(b / 4 a)^{1 / 3} y\right), Z_{1}^{\prime}(x)>0$ and $x \in\left((b / 4 a)^{1 / 3} y,+\infty\right), Z_{1}^{\prime}(x)<0$. With the sufficient condition of extreme value, $x=(b / 4 a)^{1 / 3} y$ is the maximum point of function $Z_{1}(x)$. Therefore, we get

$$
\begin{aligned}
Z_{1}(x) & \leq-a\left[\left(\frac{b}{4 a}\right)^{1 / 3} y\right]^{4}+b\left[\left(\frac{b}{4 a}\right)^{1 / 3} y\right] y^{3} \\
& =\left(4^{-1 / 3}-4^{-4 / 3}\right) a^{-1 / 3} b^{4 / 3} y^{4} .
\end{aligned}
$$

We now prove (9). Similarly, letting $Z_{2}(x)=-a x^{4}+b x^{3} y$, where $y$ is a parameter, we have

$$
Z_{2}^{\prime}(x)=-4 a x^{3}+3 b x^{2} y=0, \quad x=\frac{3 b y}{4 a} \text { or } x=0 .
$$

Obviously, for any $x \in(-\infty, 3 b y / 4 a), Z_{2}^{\prime}(x)>0$ and $x \in$ $(3 b y / 4 a,+\infty), Z_{2}^{\prime}(x)<0$. With the sufficient condition of extreme value, $x=3 b y / 4 a$ is the maximum point of function $Z_{2}(x)$. Therefore, we obtain

$$
\begin{aligned}
Z_{2}(x) & \leq-a\left(\frac{3 b y}{4 a}\right)^{4}+b\left(\frac{3 b y}{4 a}\right)^{3} y \\
& =\left[\left(\frac{3}{4}\right)^{3}-\left(\frac{3}{4}\right)^{4}\right] b^{4} a^{-3} y^{4}
\end{aligned}
$$

Lemma 8 (Cauchy-Schwartz's inequality). For any vector $x=$ $\left(x_{1}, x_{2}, \ldots, x_{n}\right) \in \mathbb{R}^{n}$ and $y=\left(y_{1}, y_{2}, \ldots, y_{n}\right) \in \mathbb{R}^{n}$, one has

$$
\begin{aligned}
& \left(x_{1} y_{1}+x_{2} y_{2}+\cdots+x_{n} y_{n}\right)^{2} \\
& \quad \leq\left(x_{1}^{2}+\cdots+x_{n}^{2}\right)\left(y_{1}^{2}+\cdots+y_{n}^{2}\right) .
\end{aligned}
$$

Lemma 9. For a sequence of numbers $a_{1}, a_{2}, \ldots, a_{n} \in \mathbb{R}_{+}$, one has

$$
\begin{aligned}
\left|a_{1}+a_{2}+\cdots+a_{n}\right| & \geq \sqrt{a_{1}^{2}+a_{2}^{2}+\cdots+a_{n}^{2}}, \\
\sqrt{\frac{a_{1}^{2}+a_{2}^{2}+\cdots+a_{n}^{2}}{n}} & \geq \frac{a_{1}+a_{2}+\cdots+a_{n}}{n} \\
& \geq \sqrt[n]{a_{1} a_{2} \cdots a_{n}} \\
& \geq \frac{n}{1 / a_{1}+1 / a_{2}+\cdots+1 / a_{n}} .
\end{aligned}
$$

Lemma 10 (see [17]). For any given real numbers $c$, $d$ and any real-valued functions $f(x, y)>0, g(x, y, z) \geq 0$, the following inequality holds:

$$
\begin{aligned}
g(x, y, z)|x|^{c}|y|^{d} & \\
\leq & \frac{c}{c+d} f(x, y)|x|^{c+d} \\
& \quad+\frac{d}{c+d}(g(x, y, z))^{(c+d) / d}(f(x, y))^{-c / d}|y|^{c+d},
\end{aligned}
$$

where $x, y, z \in \mathbb{R}$. Particularly when one takes $f(x, y)=$ $g(x, y, z)=1, c=3$, and $d=4$, then the inequality will become

$$
x^{3} y \leq \frac{3}{4} x^{4}+\frac{1}{4} y^{4}
$$

\section{The Output Feedback Model and Control Design}

In this section, we will design a linear observer system for a class of stochastic nonlinear systems with time-varying delays. Using the backstepping method, a simple linear controller will be constructed to guarantee that the closedloop stochastic system is globally asymptotically stable in probability.

Consider the following stochastic nonlinear system with time-varying delay:

$$
\begin{aligned}
& d x_{1}=\left(x_{2}+f_{1}\left(t, \bar{x}_{1}, \bar{x}_{1}(t-d(t))\right)\right) d t \\
& +g_{1}^{T}\left(t, \bar{x}_{1}, \bar{x}_{1}(t-d(t))\right) d w, \\
& d x_{2}=\left(x_{3}+f_{2}\left(t, \bar{x}_{2}, \bar{x}_{2}(t-d(t))\right)\right) d t \\
& +g_{2}^{T}\left(t, \bar{x}_{2}, \bar{x}_{2}(t-d(t))\right) d w, \\
& d x_{n}=\left(u+f_{n}\left(t, \bar{x}_{n}, \bar{x}_{n}(t-d(t))\right)\right) d t \\
& +g_{n}^{T}\left(t, \bar{x}_{n}, \bar{x}_{n}(t-d(t))\right) d w, \\
& y=x_{1},
\end{aligned}
$$

where the initial data $\{x(\theta):-d \leq \theta \leq 0\}=\xi \epsilon$ $\mathscr{C}_{\mathscr{F}_{0}}^{b}\left([-d, 0] ; \mathbb{R}^{n}\right), d(t): \mathbb{R}_{+} \rightarrow[0, d]$ is a Borel measurable 
function and $d^{\prime}(t) \leq d^{\prime}<1 ; \omega$ is an $r$-dimensional standard Wiener process defined on the complete probability space $\left(\Omega, \mathscr{F},\left\{\mathscr{F}_{t}\right\}_{t \geq 0}, P\right) . \bar{x}_{i}=\left(x_{1}, \ldots, x_{i}\right)$ is the system state, $\bar{x}_{i}(t-$ $d(t))=\left(x_{1}(t-d(t)), \ldots, x_{i}(t-d(t))\right)$ is the time-delayed state vectors, $u \in \mathbb{R}$ is the system input, and $y \in \mathbb{R}$ is the system output. $f_{i}: \mathbb{R}_{+} \times \mathbb{R}^{i} \times \mathbb{R}^{i} \rightarrow \mathbb{R}$ and $g_{i}: \mathbb{R}_{+} \times \mathbb{R}^{i} \times \mathbb{R}^{i} \rightarrow$ $\mathbb{R}^{r}$ are assumed to be locally Lipschitz in $(x(t), x(t-d(t)))$ uniformly in $t$ with $f_{i}(t, 0,0)=0, g_{i}(t, 0,0)=0, i=1, \ldots$, $n$.

Remark 11. It should be pointed out that Zhai and Zha [23] considered the global adaptive output feedback control for system (18) without the diffusion terms. And Duan and Xie [24] discussed the globally asymptotic stability for system (18) without time-varying delay. Compared with these works, we focus on the output feedback stabilization of stochastic nonlinear systems with time-varying delay (see system (18)).

Assumption 12. There exist nonnegative constants $l_{1}, l_{2} \in \mathbb{R}_{+}$ such that

$$
\begin{gathered}
\left|f_{i}\left(t, \bar{x}_{i}, \bar{x}_{i}(t-\theta(t))\right)\right| \leq l_{1}\left(\left|x_{1}\right|+\cdots+\left|x_{i}\right|\right. \\
\left.+\left|x_{1}(t-\theta(t))\right|+\cdots+\left|x_{i}(t-\theta(t))\right|\right), \\
\left|g_{i}\left(t, \bar{x}_{i}, \bar{x}_{i}(t-\theta(t))\right)\right| \leq l_{2}\left(\left|x_{1}\right|+\cdots+\left|x_{i}\right|\right. \\
\left.+\left|x_{1}(t-\theta(t))\right|+\cdots+\left|x_{i}(t-\theta(t))\right|\right) .
\end{gathered}
$$

The linear observer system is designed as

$$
\begin{gathered}
\dot{\hat{x}}_{1}=\hat{x}_{2}(t)+L a_{1}\left(x_{1}-\widehat{x}_{1}\right), \\
\dot{\hat{x}}_{2}=\widehat{x}_{3}(t)+L^{2} a_{2}\left(x_{1}-\widehat{x}_{1}\right), \\
\vdots \\
\dot{\hat{x}}_{n-1}=\hat{x}_{n}(t)+L^{n-1} a_{n-1}\left(x_{1}-\widehat{x}_{1}\right), \\
\dot{\hat{x}}_{n}=u+L^{n} a_{n}\left(x_{1}-\widehat{x}_{1}\right),
\end{gathered}
$$

where $L \geq 1$ is a constant to be determined and $a_{i}>0, i=$ $1, \ldots, n$, are coefficients of the Hurwitz polynomial

$$
p(t)=t^{n}+a_{1} t^{n-1}+\cdots+a_{n-1} t+a_{n} .
$$

The observation error $\varepsilon_{i}=\left(x_{i}-\widehat{x}_{i}\right) / L^{i-1}$ satisfies

$$
\begin{aligned}
d \varepsilon=L\left[\begin{array}{ccccc}
-a_{1} & 1 & 0 & \cdots & 0 \\
-a_{2} & 0 & 1 & \cdots & 0 \\
\vdots & \vdots & \vdots & \ddots & \vdots \\
-a_{n-1} & 0 & 0 & \cdots & 0 \\
-a_{n} & 0 & 0 & \cdots & 0
\end{array}\right] \varepsilon d t+\left[\begin{array}{c}
f_{1} \\
\frac{f_{2}}{L} \\
\vdots \\
\frac{f_{n-1}}{L^{n-2}} \\
\frac{f_{n}}{L^{n-1}}
\end{array}\right] d t \\
+\left[\begin{array}{c}
g_{1}^{T} \\
\frac{g_{2}^{T}}{L} \\
\vdots \\
\frac{g_{n-1}^{T}}{L^{n-2}} \\
\frac{g_{n}^{T}}{L^{n-1}}
\end{array}\right] d w=L A \varepsilon d t+F d t+G d w,
\end{aligned}
$$

where $A$ is a Hurwitz matrix. Therefore, there is a positivedefinite matrix $P$ such that

$$
A^{T} P+P A=-I .
$$

Theorem 13. Suppose Assumption 12 holds. Then, the equilibrium at origin of closed-loop stochastic nonlinear system (18) and (20) with the linear controller (50) below is globally asymptotically stable in probability and $P\left\{\lim _{t \rightarrow \infty}|x(t)|=0\right\}=$ 1. Furthermore, the closed-loop system has a unique solution on $[-d, \infty)$ for each $\xi \in \mathbb{R}^{n}$.

Proof. Note $z=(x, \widehat{x}), x_{i}(t)=x_{i}$, and $\hat{x}_{i}(t)=\hat{x}_{i}$ for simplicity. Consider the following Lyapunov function: $V_{0}=$ $((n+1) / 2)\left(\varepsilon^{T} P \varepsilon\right)^{2}$. Then by Lemma 10 , a direct computation yields

$$
\begin{aligned}
\mathscr{L} V_{0}= & (n+1)\left(\varepsilon^{T} P \varepsilon\right)\left(L \varepsilon^{T}\left(A^{T} P+P A\right) \varepsilon+2 \varepsilon^{T} P F\right) \\
& +\frac{1}{2} \operatorname{tr}\left(G^{T}(n+1)\left(4 P \varepsilon \varepsilon^{T} P+2 \varepsilon^{T} P \varepsilon P\right) G\right) \\
\leq & -(n+1) \lambda_{\min } L|\varepsilon|^{4}+2(n+1) \lambda_{\max }^{2}|\varepsilon|^{3}|F| \\
& +3(n+1) r \sqrt{r} \lambda_{\max }^{2}|\varepsilon|^{2}|G|^{2} \\
\leq & -(n+1) \lambda_{\min } L|\varepsilon|^{4} \\
& +\frac{3(n+1)}{2}(1+r \sqrt{r}) \lambda_{\max }^{2}|\varepsilon|^{4} \\
& +\frac{n+1}{2} \lambda_{\max }^{2}|F|^{4}+\frac{3(n+1)}{2} r \sqrt{r} \lambda_{\max }^{2}|G|^{4},
\end{aligned}
$$

where $\lambda_{\min }$ denotes the minimum eigenvalue of the matrix $P$ and $\lambda_{\max }$ denotes the maximum eigenvalue of the matrix $P$. 
By Lemmas 8 and 9 and Assumption 12, one gets

$$
\begin{aligned}
|F|^{4} & \leq l_{1}^{4} n^{5}\left(x_{1}^{4}+\frac{1}{L^{4}} x_{2}^{4}+\cdots+\frac{1}{L^{4 n-4}} x_{n}^{4}\right) \\
+ & l_{1}^{4} n^{5}\left(x_{1}^{4}(t-\theta(t))+\frac{1}{L^{4}} x_{2}^{4}(t-\theta(t))+\cdots\right. \\
+ & \left.\frac{1}{L^{4 n-4}} x_{n}^{4}(t-\theta(t))\right), \\
|G|^{4} & \leq l_{2}^{4} n^{5}\left(x_{1}^{4}+\frac{1}{L^{4}} x_{2}^{4}+\cdots+\frac{1}{L^{4 n-4}} x_{n}^{4}\right) \\
+ & l_{2}^{4} n^{5}\left(x_{1}^{4}(t-\theta(t))+\frac{1}{L^{4}} x_{2}^{4}(t-\theta(t))+\cdots\right. \\
& \left.+\frac{1}{L^{4 n-4}} x_{n}^{4}(t-\theta(t))\right) .
\end{aligned}
$$

Substituting (25) into (24), we have

$$
\begin{aligned}
\mathscr{L} V_{0} \leq & -(n+1) \lambda_{\min } L|\varepsilon|^{4} \\
& +\frac{3(n+1)}{2}(1+r \sqrt{r}) \lambda_{\max }^{2}|\varepsilon|^{4} \\
& +\frac{(n+1) n^{5}}{2} \lambda_{\max }^{2}\left(l_{1}^{4}+3 r \sqrt{r} l_{2}^{4}\right) \sum_{i=1}^{n} \frac{x_{i}^{4}\left(t-\theta_{t}\right)}{L^{4 i-4}} \\
& +\frac{(n+1) n^{5}}{2} \lambda_{\max }^{2}\left(l_{1}^{4}+3 r \sqrt{r} l_{2}^{4}\right) \sum_{i=1}^{n} \frac{x_{i}^{4}}{L^{4 i-4}} \\
= & -(n+1) \lambda_{\min } L|\varepsilon|^{4} \\
& +\frac{3(n+1)}{2}(1+r \sqrt{r}) \lambda_{\max }^{2}|\varepsilon|^{4}+\alpha_{1} \sum_{i=1}^{n} \frac{x_{i}^{4}}{L^{4 i-4}} \\
& +\alpha_{1} \sum_{i=1}^{n} \frac{x_{i}^{4}(t-d(t))}{L^{4 i-4}}
\end{aligned}
$$

where

$$
\alpha_{1}=\frac{(n+1) n^{5}}{2} \lambda_{\max }^{2}\left(l_{1}^{4}+3 r \sqrt{r} l_{2}^{4}\right)
$$

Take

$$
W=\frac{\alpha_{1}}{1-d^{\prime}} \sum_{i=1}^{n} \int_{t-d(t)}^{t} \frac{x_{i}^{4}(s)}{L^{4 i-4}} d s
$$

then

$$
\begin{aligned}
& \mathscr{L}\left(V_{0}+W\right) \\
& \leq-\left((n+1) \lambda_{\min } L-\frac{3(n+1)}{2}(1+r \sqrt{r}) \lambda_{\max }^{2}\right)|\varepsilon|^{4} \\
&+\alpha_{1}\left(1+\frac{1}{1-d^{\prime}}\right) \sum_{i=1}^{n} \frac{x_{i}^{4}(t)}{L^{4 i-4}} .
\end{aligned}
$$

Substituting $x_{i}=\widehat{x}_{i}+L^{i-1} \varepsilon_{i}, i=1, \ldots, n$, into (29), one gets

$$
\begin{aligned}
\mathscr{L}\left(V_{0}+W\right) \leq & -\left((n+1) \lambda_{\min } L-c_{0}\right)|\varepsilon|^{4} \\
& +c_{1} \sum_{i=1}^{n} \frac{\widehat{x}_{i}^{4}}{L^{4 i-4}},
\end{aligned}
$$

where

$$
\begin{aligned}
& c_{0}=\frac{3(n+1)}{2}(1+r \sqrt{r}) \lambda_{\max }^{2}+8 n \alpha_{1}\left(1+\frac{1}{1-d^{\prime}}\right), \\
& c_{1}=8 \alpha_{1}\left(1+\frac{1}{1-d^{\prime}}\right) .
\end{aligned}
$$

Next, we will combine the backstepping design method with the mathematical induction to design the linear output feedback control.

Step 1. Construct the Lyapunov function $V_{1}=V_{0}+W+$ $(1 / 4) \widehat{x}_{1}^{4}$. A direct calculation gives

$$
\begin{aligned}
\mathscr{L} V_{1}= & \mathscr{L} V_{0}+\mathscr{L} W+\widehat{x}_{1}^{3}\left(\widehat{x}_{2}+L a_{1} \varepsilon_{1}\right) \\
\leq & -\left((n+1) \lambda_{\min } L-c_{0}\right)|\varepsilon|^{4}+c_{1} \sum_{i=1}^{n} \frac{\widehat{x}_{i}^{4}}{L^{4 i-4}}+\widehat{x}_{1}^{3} \widehat{x}_{2} \\
& +L a_{1} \widehat{x}_{1}^{3} \varepsilon_{1} \\
\leq & -\left(n \lambda_{\min } L-c_{0}\right)|\varepsilon|^{4}+c_{1} \sum_{i=1}^{n} \frac{\widehat{x}_{i}^{4}}{L^{4 i-4}} \\
& +k_{1} \lambda_{\min }^{-1 / 3} a_{1}^{4 / 3} L \widehat{x}_{1}^{4}+\widehat{x}_{1}^{3} \widehat{x}_{2},
\end{aligned}
$$

where $k_{1}$ is defined in Lemma 7.

Define $\xi_{2}=\widehat{x}_{2}-\widehat{x}_{2}^{*}$ with $\widehat{x}_{2}^{*}$ being a virtual control. Then, we have

$$
\begin{aligned}
\mathscr{L} V_{1} \leq & -\left(n \lambda_{\min } L-c_{0}\right)|\varepsilon|^{4}+c_{1}\left(\frac{\widehat{x}_{3}^{4}}{L^{8}}+\cdots+\frac{\widehat{x}_{n}^{4}}{L^{4 n-4}}\right) \\
& +\left(c_{1}+k_{1} \lambda_{\min }^{-1 / 3} a_{1}^{4 / 3} L\right) \hat{x}_{1}^{4}+\frac{c_{1}}{L^{4}}\left(\xi_{2}+\widehat{x}_{2}^{*}\right)^{4} \\
& +\widehat{x}_{1}^{3}\left(\xi_{2}+\widehat{x}_{2}^{*}\right) \\
\leq & -\left(n \lambda_{\min } L-c_{0}\right)|\varepsilon|^{4}+c_{1}\left(\frac{\widehat{x}_{3}^{4}}{L^{8}}+\cdots+\frac{\widehat{x}_{n}^{4}}{L^{4 n-4}}\right) \\
& +\left(c_{1}+k_{1} \lambda_{\min }^{-1 / 3} a_{1}^{4 / 3} L\right) \hat{x}_{1}^{4}+\frac{8 c_{1}}{L^{4}} \xi_{2}^{4}+\frac{8 c_{1}}{L^{4}}\left(\hat{x}_{2}^{*}\right)^{4} \\
& +\widehat{x}_{1}^{3} \xi_{2}+\widehat{x}_{1}^{3} \hat{x}_{2}^{*} .
\end{aligned}
$$

Choose the virtual controller

$$
\widehat{x}_{2}^{*}=-L\left(n+c_{1}+k_{1} \lambda_{1}^{-1 / 3} a_{1}^{4 / 3}\right) \widehat{x}_{1}=-L b_{1} \widehat{x}_{1},
$$

where $b_{1}>0$ is independent of $L$. Hence,

$$
\begin{aligned}
\mathscr{L} V_{1} \leq & -\left(n \lambda_{\min } L-c_{0}\right)|\varepsilon|^{4}+c_{1}\left(\frac{\widehat{x}_{3}^{4}}{L^{8}}+\cdots+\frac{\widehat{x}_{n}^{4}}{L^{4 n-4}}\right) \\
& +\left(8 c_{1} b_{1}^{4}-n L\right) \hat{x}_{1}^{4}+\frac{8 c_{1}}{L^{4}} \xi_{2}^{4}+\widehat{x}_{1}^{3} \xi_{2} .
\end{aligned}
$$


Step $k$. Suppose that, at Step $k$, there exists a smooth Lyapunov function $V_{k}$ which is positive definite, radially unbounded, and twice continuously differentiable, satisfying

$$
\begin{aligned}
\mathscr{L} V_{k} \leq & -\left((n+1-k) \lambda_{\min } L-c_{0}\right)|\varepsilon|^{4} \\
& -\sum_{j=1}^{k} \frac{1}{L^{4 j-4}}\left((n+1-k) L-8 c_{1} b_{j}^{4}\right) \xi_{j}^{4} \\
& +c_{1}\left(\frac{1}{L^{4 k+4}} \widehat{x}_{k+2}^{4}+\cdots+\frac{1}{L^{4 n-4}} \widehat{x}_{n}^{4}\right)+\frac{8 c_{1}}{L^{4 k}} \xi_{k+1}^{4} \\
& +\frac{1}{L^{4 k-4}} \xi_{k}^{3} \xi_{k+1},
\end{aligned}
$$

with a set of virtual controllers $\widehat{x}_{1}^{*}, \hat{x}_{2}^{*}, \ldots, \hat{x}_{k}^{*}$, defined by $\widehat{x}_{1}^{*}=$ $0, \widehat{x}_{i}^{*}=-L b_{i-1} \xi_{i-1}$ with $b_{i-1}>0$ being independent of the gain constant $L$, where $\xi_{i}=\widehat{x}_{i}-\widehat{x}_{i}^{*}, i=1, \ldots, n$.

From the above definition, we have

$$
\begin{aligned}
\xi_{k+1}= & \widehat{x}_{k+1}+L b_{k} \xi_{k} \\
= & \widehat{x}_{k+1}+L b_{k} \widehat{x}_{k}+L^{2} b_{k} b_{k-1} \widehat{x}_{k-1}+\cdots \\
& +L^{k} b_{k} b_{k-1} \cdots b_{1} \widehat{x}_{1} .
\end{aligned}
$$

A direct computation yields

$$
\begin{aligned}
& \mathscr{L}\left(\frac{1}{4 L^{4 k}} \xi_{k+1}^{4}\right)=\frac{1}{L^{4 k}} \xi_{k+1}^{3}\left(\widehat{x}_{k+2}+L^{k+1} a_{k+1} \varepsilon_{1}\right. \\
& \left.\quad+L b_{k} \sum_{i=1}^{k} \frac{\partial \xi_{k}}{\partial x_{i}}\left(\widehat{x}_{i+1}+L^{i} a_{i} \varepsilon_{1}\right)\right)=\frac{1}{L^{4 k}} \xi_{k+1}^{3}\left(\widehat{x}_{k+2}\right. \\
& \quad+L^{k+1} a_{k+1} \varepsilon_{1} \\
& \left.\quad+\sum_{i=1}^{k} L^{k-i+1} b_{k} \cdots b_{i}\left(\xi_{i+1}-L b_{i} \xi_{i}+L^{i} a_{i} \varepsilon_{1}\right)\right)=\frac{1}{L^{4 k}} \\
& \quad+\xi_{k+1}^{3}\left(\widehat{x}_{k+2}+L^{k+1} d_{0} \varepsilon_{1}+L^{k+1} d_{1} \xi_{1}+L^{k} d_{2} \xi_{2}+\cdots\right. \\
& \left.\quad+L^{2} d_{k} \xi_{k}+L d_{k+1} \xi_{k+1}\right),
\end{aligned}
$$

where

$$
\begin{gathered}
d_{0}=a_{k+1}+\left(b_{k} \cdots b_{1} a_{1}\right)+\left(b_{k} \cdots b_{2} a_{2}\right)+\cdots+b_{k} a_{k} \\
d_{1}=-b_{k} \cdots b_{1}^{2} \\
d_{2}=b_{k} \cdots b_{1}-b_{k} \cdots b_{2}^{2} \\
\vdots \\
d_{k}=b_{k} b_{k-1}-b_{k}^{2}, \\
d_{k+1}=b_{k}>0 .
\end{gathered}
$$

Obviously, $d_{0}, d_{1}, \ldots, d_{k+1}$ are suitable real numbers and they are independent of the gain constant $L$.
Let us consider the following Lyapunov function:

$$
V_{k+1}=V_{k}+\frac{1}{4 L^{4 k}} \xi_{k+1}^{4}
$$

Then, combining (36) and (38), we obtain

$$
\begin{aligned}
& \mathscr{L} V_{k+1} \leq-\left((n+1-k) \lambda_{\min } L-c_{0}\right)|\varepsilon|^{4} \\
& -\sum_{j=1}^{k} \frac{1}{L^{4 j-4}}\left((n+1-k) L-8 c_{1} b_{j}^{4}\right) \xi_{j}^{4} \\
& +c_{1}\left(\frac{1}{L^{4 k+4}} \widehat{x}_{k+2}^{4}+\cdots+\frac{1}{L^{4 n-4}} \widehat{x}_{n}^{4}\right)+\frac{8 c_{1}}{L^{4 k}} \xi_{k+1}^{4} \\
& +\frac{1}{L^{4 k-4}} \xi_{k}^{3} \xi_{k+1}+\frac{1}{L^{4 k}} \xi_{k+1}^{3}\left(\widehat{x}_{k+2}+L^{k+1} d_{0} \varepsilon_{1}\right. \\
& \left.+L^{k+1} d_{1} \xi_{1}+\cdots+L^{2} d_{k} \xi_{k}+L d_{k+1} \xi_{k+1}\right) \\
& \leq-\left((n-k) \lambda_{\min } L-c_{0}\right)|\varepsilon|^{4} \\
& -\sum_{j=1}^{k} \frac{1}{L^{4 j-4}}\left((n-k) L-8 c_{1} b_{j}^{4}\right) \xi_{j}^{4}+c_{1}\left(\frac{1}{L^{4 k+8}} \widehat{x}_{k+3}^{4}\right. \\
& \left.+\cdots+\frac{1}{L^{4 n-4}} \widehat{x}_{n}^{4}\right)+\frac{8 c_{1}}{L^{4 k+4}} \xi_{k+2}^{4}+\frac{8 c_{1}}{L^{4 k+4}}\left(\widehat{x}_{k+2}^{*}\right)^{4} \\
& +\frac{1}{L^{4 k}} \xi_{k+1}^{3} \widehat{x}_{k+2}^{*}+\frac{1}{L^{4 k}} \xi_{k+1}^{3} \xi_{k+2}+\frac{d_{k+1}+8\left(c_{1} / L\right)}{L^{4 k-1}} \\
& \cdot \xi_{k+1}^{4}+\left(-\lambda_{\min } L \varepsilon_{1}^{4}+\frac{1}{L^{3 k-1}} d_{0} \xi_{k+1}^{3} \varepsilon_{1}\right)+\left(-L \xi_{1}^{4}\right. \\
& \left.+\frac{d_{1}}{L^{3 k-1}} \xi_{k+1}^{3} \xi_{1}\right)+\left(-\frac{1}{L^{3}} \xi_{2}^{4}+\frac{d_{2}}{L^{3 k}} \xi_{k+1}^{3} \xi_{2}\right)+\cdots \\
& +\left(-\frac{1}{L^{4 k-9}} \xi_{k-1}^{4}+\frac{d_{k-1}}{L^{4 k-3}} \xi_{k+1}^{3} \xi_{k-1}\right)+\left(-\frac{1}{L^{4 k-5}} \xi_{k}^{4}\right. \\
& \left.+\frac{d_{k}}{L^{4 k-2}} \xi_{k+1}^{3} \xi_{k}+\frac{1}{L^{4 k-4}} \xi_{k}^{3} \xi_{k+1}\right) .
\end{aligned}
$$

It follows from Lemma 7 that

$$
\begin{gathered}
-\lambda_{\min } L \varepsilon_{1}^{4}+\frac{1}{L^{3 k-1}} d_{0} \xi_{k+1}^{3} \varepsilon_{1} \leq k_{1} d_{0}^{4 / 3} \lambda_{\min }^{-1 / 3} \frac{\xi_{k+1}^{4}}{L^{4 k-1}} \\
-L \xi_{1}^{4}+\frac{d_{1}}{L^{3 k-1}} \xi_{k+1}^{3} \xi_{1} \leq k_{1} d_{1}^{4 / 3} \frac{\xi_{k+1}^{4}}{L^{4 k-1}} \\
-\frac{1}{L^{3}} \xi_{2}^{4}+\frac{d_{2}}{L^{3 k}} \xi_{k+1}^{3} \xi_{2} \leq k_{1} d_{2}^{4 / 3} \frac{\xi_{k+1}^{4}}{L^{4 k-1}} \\
\vdots \\
-\frac{1}{L^{4 k-9}} \xi_{k-1}^{4}+\frac{d_{k-1}}{L^{4 k-3}} \xi_{k+1}^{3} \xi_{k-1} \leq k_{1} d_{k-1}^{4 / 3} \frac{\xi_{k+1}^{4}}{L^{4 k-1}}
\end{gathered}
$$


In particular, the last term of (41) yields

$$
\begin{aligned}
- & \frac{1}{L^{4 k-5}} \xi_{k}^{4}+\frac{d_{k}}{L^{4 k-2}} \xi_{k+1}^{3} \xi_{k}+\frac{1}{L^{4 k-4}} \xi_{k}^{3} \xi_{k+1} \\
\leq & {\left[-\frac{1}{2 L^{4 k-5}} \xi_{k}^{4}+\frac{d_{k}}{L^{4 k-2}} \xi_{k+1}^{3} \xi_{k}\right] } \\
& +\left[-\frac{1}{2 L^{4 k-5}} \xi_{k}^{4}+\frac{1}{L^{4 k-4}} \xi_{k}^{3} \xi_{k+1}\right] \\
\leq & \left(k_{1}\left(\frac{1}{2}\right)^{-1 / 3} d_{k}^{4 / 3}+k_{2}\left(\frac{1}{2}\right)^{-3}\right) \frac{\xi_{k+1}^{4}}{L^{4 k-1}} .
\end{aligned}
$$

Hence,

$$
\begin{aligned}
\mathscr{L} V_{k+1} \leq & -\left((n-k) \lambda_{\min } L-c_{0}\right)|\varepsilon|^{4} \\
& -\sum_{j=1}^{k} \frac{1}{L^{4 j-4}}\left((n-k) L-8 c_{1} b_{j}^{4}\right) \xi_{j}^{4} \\
& +c_{1}\left(\frac{1}{L^{4 k+8}} \widehat{x}_{k+3}^{4}+\cdots+\frac{1}{L^{4 n-4}} \widehat{x}_{n}^{4}\right) \\
& +\frac{8 c_{1}}{L^{4 k+4}} \xi_{k+2}^{4}+\frac{8 c_{1}}{L^{4 k+4}}\left(\hat{x}_{k+2}^{*}\right)^{4} \\
& +\frac{1}{L^{4 k}} \xi_{k+1}^{3} \widehat{x}_{k+2}^{*}+\frac{1}{L^{4 k}} \xi_{k+1}^{3} \xi_{k+2} \\
& +\frac{1}{L^{4 k-1}}\left(d_{0}^{\prime}+d_{1}^{\prime}+\cdots+d_{k+1}^{\prime}\right) \xi_{k+1}^{4},
\end{aligned}
$$

where

$$
\begin{gathered}
d_{0}^{\prime}=k_{1} d_{0}^{4 / 3} \lambda_{\mathrm{min}}^{-1 / 3}, \\
d_{1}^{\prime}=k_{1} d_{1}^{4 / 3}, \\
\vdots \\
d_{k-1}^{\prime}=k_{1} d_{k-1}^{4 / 3}, \\
d_{k}^{\prime}=k_{1}\left(\frac{1}{2}\right)^{-1 / 3} d_{k}^{4 / 3}+k_{2}\left(\frac{1}{2}\right)^{-3}, \\
d_{k+1}^{\prime}=d_{k+1}+8 c_{1} .
\end{gathered}
$$

Now, we choose the following linear controller:

$$
\begin{aligned}
\hat{x}_{k+2}^{*} & =-L\left(n-k+d_{0}^{\prime}+d_{1}^{\prime}+\cdots+d_{k+1}^{\prime}\right) \xi_{k+1} \\
& =-L b_{k+1} \xi_{k+1},
\end{aligned}
$$

with $b_{k+1}>0$ being independent of $L$.
So it follows from (44) that

$$
\begin{aligned}
\mathscr{L} V_{k+1} \leq & -\left((n-k) \lambda_{\min } L-c_{0}\right)|\varepsilon|^{4} \\
& -\sum_{j=1}^{k+1} \frac{1}{L^{4 j-4}}\left((n-k) L-8 c_{1} b_{j}^{4}\right) \xi_{j}^{4} \\
& +c_{1}\left(\frac{1}{L^{4 k+8}} \widehat{x}_{k+3}^{4}+\cdots+\frac{1}{L^{4 n-4}} \widehat{x}_{n}^{4}\right) \\
& +\frac{8 c_{1}}{L^{4 k+4}} \xi_{k+2}^{4}+\frac{1}{L^{4 k}} \xi_{k+1}^{3} \xi_{k+2} .
\end{aligned}
$$

Step $n$. Using the inductive argument step by step, at the $(n-$ 1)th step, we get

$$
\begin{aligned}
\mathscr{L} V_{n-1} \leq & -\left(2 \lambda_{\min } L-c_{0}\right)|\varepsilon|^{4} \\
& -\sum_{j=1}^{n-1} \frac{1}{L^{4 j-4}}\left(2 L-8 c_{1} b_{j}^{4}\right) \xi_{j}^{4}+\frac{8 c_{1}}{L^{4 n-4}} \xi_{n}^{4} \\
& +\frac{1}{L^{4 n-8}} \xi_{n-1}^{3} \xi_{n} .
\end{aligned}
$$

Noting the function $V_{n}=V_{n-1}+\left(1 / 4 L^{4 n-4}\right) \xi_{n}^{4}$, we have

$$
\begin{aligned}
& \mathscr{L} V_{n} \leq-\left(2 \lambda_{\min } L-c_{0}\right)|\varepsilon|^{4} \\
& -\sum_{j=1}^{n-1} \frac{1}{L^{4 j-4}}\left(2 L-8 c_{1} b_{j}^{4}\right) \xi_{j}^{4}+\frac{8 c_{1}}{L^{4 n-4}} \xi_{n}^{4}+\frac{1}{L^{4 n-8}} \\
& \cdot \xi_{n-1}^{3} \xi_{n}+\frac{1}{L^{4 n-4}} \xi_{n}^{3} \widehat{x}_{n+1}+\frac{1}{L^{4 n-4}}\left(L^{n} d_{0} \varepsilon_{1}+L^{n} d_{1} \xi_{1}\right. \\
& \left.+\cdots+L^{2} d_{n-1} \xi_{n-1}+L d_{n} \xi_{n}\right) \xi_{n}^{3} \leq-\left(\lambda_{\min } L-c_{0}\right) \\
& \cdot|\varepsilon|^{4}-\sum_{j=1}^{n-1} \frac{1}{L^{4 j-4}}\left(L-8 c_{1} b_{j}^{4}\right) \xi_{j}^{4}+\frac{1}{L^{4 n-4}} \xi_{n}^{3} \widehat{x}_{n+1} \\
& +\left(\frac{8 c_{1}}{L^{4 n-4}}+\frac{1}{L^{4 n-5}} d_{n}\right) \xi_{n}^{4}+\frac{1}{L^{4 n-5}}\left(d_{0}^{\prime}+d_{1}^{\prime}+\cdots\right. \\
& \left.+d_{n-1}^{\prime}\right) \xi_{n}^{4} \leq-\left(\lambda_{\min } L-c_{0}\right)|\varepsilon|^{4} \\
& +\sum_{j=1}^{n-1} \frac{1}{L^{4 j-4}}\left(L-8 c_{1} b_{j}^{4}\right) \xi_{j}^{4}+\frac{1}{L^{4 n-4}} \xi_{n}^{3} \widehat{x}_{n+1} \\
& +\frac{1}{L^{4 n-5}}\left(d_{0}^{\prime}+d_{1}^{\prime}+\cdots+d_{n-1}^{\prime}+8 c_{1}+d_{n}\right) \xi_{n}^{4} .
\end{aligned}
$$

We now design the linear controller

$$
\begin{aligned}
u= & \widehat{x}_{n+1}=-L b_{n} \xi_{n}=-L b_{n}\left(\widehat{x}_{n}+L b_{n-1} \widehat{x}_{n-1}\right. \\
& \left.+L^{2} b_{n-1} b_{n-2} \widehat{x}_{n-2}+\cdots+L^{n-1} b_{n-1} b_{n-2} \cdots b_{1} \widehat{x}_{1}\right)
\end{aligned}
$$

where $b_{n}=1+d_{0}^{\prime}+d_{1}^{\prime}+\cdots+d_{n-1}^{\prime}+8 c_{1}+d_{n}>0$ is a real number independent of the gain parameter $L$. Thus,

$$
\mathscr{L} V_{n} \leq-\alpha_{3}\left(\varepsilon, \xi_{1}, \ldots, \xi_{n}\right),
$$


where $\alpha_{3}\left(\varepsilon, \xi_{1}, \ldots, \xi_{n}\right):=\left(\lambda_{\min } L-c_{0}\right)|\varepsilon|^{4}+\sum_{j=1}^{n-1}\left(1 / L^{4 j-4}\right)(L-$ $\left.8 c_{1} b_{j}^{4}\right) \xi_{j}^{4}+\left(1 / L^{4 n-5}\right) \xi_{n}^{4}$.

Choosing the gain constant $L=\max \left\{1, c_{0} / \lambda_{\min }\right.$, $\left.8 c_{1} b_{1}^{4}, \ldots, 8 c_{1} b_{n-1}^{2}\right\}$, it is obvious that $\alpha_{3}\left(\varepsilon, \xi_{1}, \ldots, \xi_{n}\right)$ is a positive-definite and proper function. Thus, we see that (6) is true.

Now, we prove (5). From the above process, we have constructed the Lyapunov function

$$
V_{n}=\bar{V}+\frac{\alpha_{1}}{1-d^{\prime}} \sum_{i=1}^{n} \int_{t-d(t)}^{t} \frac{x_{i}^{4}(s)}{L^{4 i-4}} d s
$$

where $\alpha_{1}$ and $d^{\prime}$ are positive parameters and

$$
\bar{V}=\frac{n+1}{2}\left(\varepsilon^{T} P \varepsilon\right)^{2}+\sum_{i=1}^{n} \frac{\xi_{i}^{4}}{4 L^{4 i-4}} .
$$

It is easy to verify that $\bar{V}$ is $\mathscr{C}^{2}$ on $z$. Suppose that $U(z)$ is continuous, positive definite, and radially unbounded. Then by Lemmas 5 and 6 , there exist positive constants $\underline{\gamma}$ and $\bar{\gamma}$ and two class $\mathscr{K}_{\infty}$ functions $\beta_{1}$ and $\alpha_{11}$ such that

$$
\begin{gathered}
\beta_{1}(|z|) \leq U(z) \leq \alpha_{11}(|z|), \\
\underline{\gamma}\|z\|_{\Delta}^{4} \leq U(z) \leq \bar{\gamma}\|z\|_{\Delta}^{4} .
\end{gathered}
$$

It follows from $d(t): \mathbb{R}_{+} \rightarrow[0, d]$ that

$$
\begin{aligned}
& \frac{\alpha_{1}}{1-d^{\prime}} \sum_{i=1}^{n} \int_{t-d(t)}^{t} \frac{x_{i}^{4}(s)}{L^{4 i-4}} d s \leq \gamma \int_{t-d(t)}^{t} \sum_{i=1}^{n} \frac{x_{i}^{4}(s)}{L^{4 i-4}} d s \\
& \leq \bar{\gamma} \int_{t-d(t)}^{t}\|z(s)\|_{\Delta}^{4} d s \leq \widetilde{\gamma} \int_{t-d(t)}^{t} \alpha_{11}(|z(s)|) d s \\
& =\widetilde{\gamma} \int_{-d(t)}^{0} \alpha_{11}(|z(\sigma+t)|) d(\sigma+t) \\
& \leq \widetilde{\gamma} \int_{-d}^{0} \alpha_{11}(|z(\sigma+t)|) d(\sigma+t) \\
& \leq \widehat{\gamma} \sup _{-d \leq \sigma \leq 0} \alpha_{11}(|z(\sigma+t)|) \\
& \leq \alpha_{22}\left(\sup _{-d \leq \sigma \leq 0}|z(\sigma+t)|\right)
\end{aligned}
$$

where $\gamma=\alpha_{1} /\left(1-d^{\prime}\right), \tilde{\gamma}$ and $\hat{\gamma}$ are positive constants, and $\alpha_{22}$ is a class $\mathscr{K}_{\infty}$ function. Noting that

$$
|z(t)| \leq \sup _{-d \leq \sigma \leq 0}|z(\sigma+t)|
$$

so

$$
\alpha_{11}(|z(t)|) \leq \alpha_{11}\left(\sup _{-d \leq \sigma \leq 0}|z(\sigma+t)|\right) .
$$

Defining

$$
\begin{aligned}
\beta_{2}\left(\sup _{-d \leq \sigma \leq 0}|z(\sigma+t)|\right) \\
:=\alpha_{11}\left(\sup _{-d \leq \sigma \leq 0}|z(\sigma+t)|\right) \\
\quad+\alpha_{22}\left(\sup _{-d \leq \sigma \leq 0}|z(\sigma+t)|\right),
\end{aligned}
$$

one gets

$$
\beta_{1}(|z(t)|) \leq V_{n}(z(t)) \leq \beta_{2}\left(\sup _{-d \leq \sigma \leq 0}|z(\sigma+t)|\right) .
$$

Therefore, by Lemma 4 , the equilibrium of closed-loop nonlinear stochastic system (18), (20), and (50) is globally asymptotically stable and the closed-loop system has a unique solution on $[-d, \infty)$ for each $\xi \in \mathbb{R}^{n}$.

Remark 14. Letting $f_{i}=0(i=1 \ldots, n)$ and $d(t) \equiv 0$ in system (18), then the model will reduce to that introduced in [9]. Therefore, our result extends and improves those given in [9].

Remark 15. It should be pointed out that, compared with the $C^{1}$ output feedback controller in [20], we see that the linear controller (50) based on the observable linearization is a smooth one.

Remark 16. In this paper, combining the backstepping design with mathematical induction, we extend the method in [21] to the stochastic nonlinear system with time-varying delay. Although the globally asymptotic stability of stochastic nonlinear system has been discussed by Liu and Zhang in [12], they did not consider the term of time-varying delay. Therefore, our conclusion is proposed for the first time.

Remark 17. In [22], Liu and Xie considered the state feedback stability of stochastic feedforward nonlinear systems with time-varying delays. They introduced the homogeneous domination approach to construct a state feedback controller. It is generally known that in real lives the output feedback control is easier to achieve than the state feedback control and has great theory significance and utility value. Therefore, in this paper we combine the backstepping method with the mathematical induction to design a Lyapunov-Krasovskii functional and construct a linear output feedback controller for stochastic nonlinear systems with time-varying delay. Moreover, we impose different restricted conditions on functions $f_{i}$ and $g_{i}(i=1, \ldots, n)$ to ensure the global stability of stochastic nonlinear systems with time-varying delays.

\section{An Example}

In this section, we will use a simulation example to illustrate our main results. 
Consider the following stochastic nonlinear system:

$$
\begin{aligned}
d x_{1}= & \left(x_{2}+\frac{1}{16} x_{1} \sin x_{2}^{2}\right) d t \\
& +\frac{1}{16} \cos \left(x_{2}(t-d(t))\right) d w, \\
d x_{2}= & u d t \\
y= & x_{1},
\end{aligned}
$$

where the time-varying delay $d(t)=\cos (t)$. Obviously, we have $\left|f_{1}(x)\right| \leq(1 / 16)\left(\left|x_{1}\right|+\left|x_{2}(t-\cos (t))\right|\right)$, $\left|g_{1}(x)\right| \leq(1 / 16)\left(\left|x_{1}\right|+\left|x_{2}(t-\cos (t))\right|\right)$, which implies that Assumption 12 holds. Moreover, both of them are locally Lipschitz. The linear observer system is designed as

$$
\begin{aligned}
& \dot{\hat{x}}_{1}=\widehat{x}_{2}+L\left(x_{1}-\widehat{x}_{1}\right), \\
& \dot{\hat{x}}_{2}=u+L^{2}\left(x_{1}-\widehat{x}_{1}\right)
\end{aligned}
$$

with a suitable choice of the parameter $L$. The observation errors

$$
\begin{aligned}
& \varepsilon_{1}=x_{1}-\widehat{x}_{1}, \\
& \varepsilon_{2}=x_{2}-\widehat{x}_{2}
\end{aligned}
$$

satisfy

$$
\begin{aligned}
d \varepsilon & =L\left[\begin{array}{ll}
-1 & 1 \\
-1 & 0
\end{array}\right] \varepsilon d t+\left[\begin{array}{l}
f_{1} \\
\frac{f_{2}}{L}
\end{array}\right] d t+\left[\begin{array}{c}
g_{1}^{T} \\
\frac{g_{2}^{T}}{L}
\end{array}\right] d w \\
& =L A \varepsilon d t+F d t+G d w .
\end{aligned}
$$

For the above matrix $A$, there exists a positive-definite matrix $P$ satisfying $A^{T} P+P A=-I$, where

$$
P=\left[\begin{array}{cc}
1 & -\frac{1}{2} \\
-\frac{1}{2} & \frac{3}{2}
\end{array}\right] \text {. }
$$

It is easy to get the corresponding eigenvalues $\lambda_{\min }=(5-$ $\sqrt{5}) / 4$ and $\lambda_{\max }=(5+\sqrt{5}) / 4$.

Consider the Lyapunov-Krasovskii functional

$$
\begin{aligned}
V= & \frac{3}{2}\left(\varepsilon^{T} P \varepsilon\right)^{2}+\frac{\alpha_{1}}{1+\sin x} \sum_{i=1}^{2} \int_{t-\cos t}^{t} \frac{x_{i}^{4}(s)}{L^{4 i-4}} d s+\frac{1}{4} \widehat{x}_{1}^{4} \\
& +\frac{1}{4 L^{4}}\left(\hat{x}_{2}+L b_{1} \widehat{x}_{1}\right)^{4}
\end{aligned}
$$

Then, we take $\alpha_{1}=0.02, b_{1}=2, b_{2}=20, L=160$, and the linear control $u=-3200\left(\hat{x}_{2}+L b_{1} \hat{x}_{1}\right)$. By Theorem 13 , we see that closed-loop system (60), (61) and the linear controller $u$ are globally asymptotically stable in probability and there is a unique solution on $[-d, \infty)$. (The state response and control input with initial conditions $x_{1}(0)=0.5, x_{2}(0)=50, \hat{x}_{1}(0)=$ 0.6 , and $\hat{x}_{2}(0)=-100$ are presented in Figures $1(\mathrm{a}), 1(\mathrm{~b})$, and 2 , where hat $x_{1}$ and hat $x_{2}$ denote $\hat{x}_{1}$ and $\hat{x}_{2}$.)

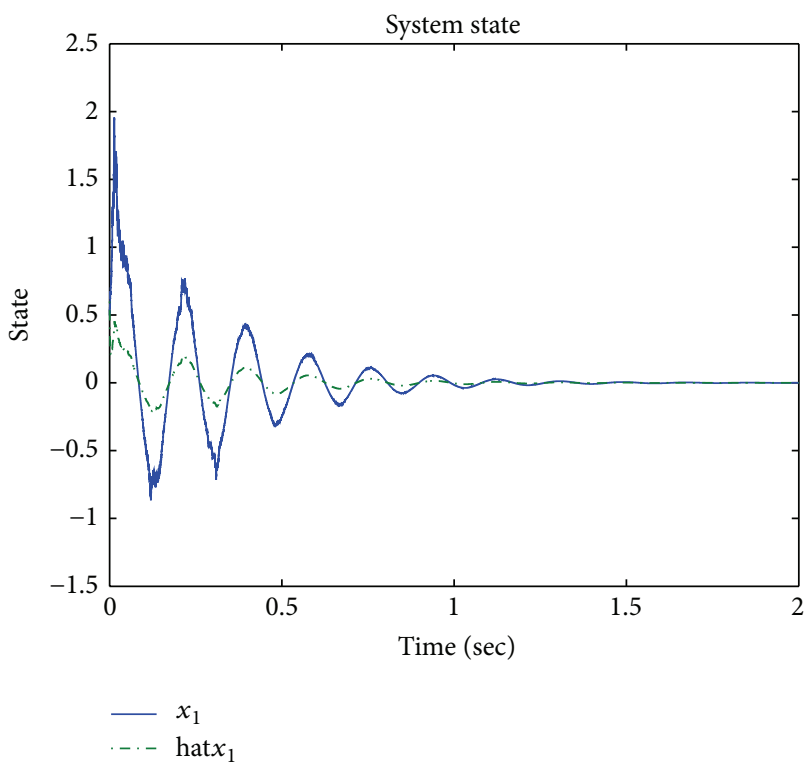

(a)

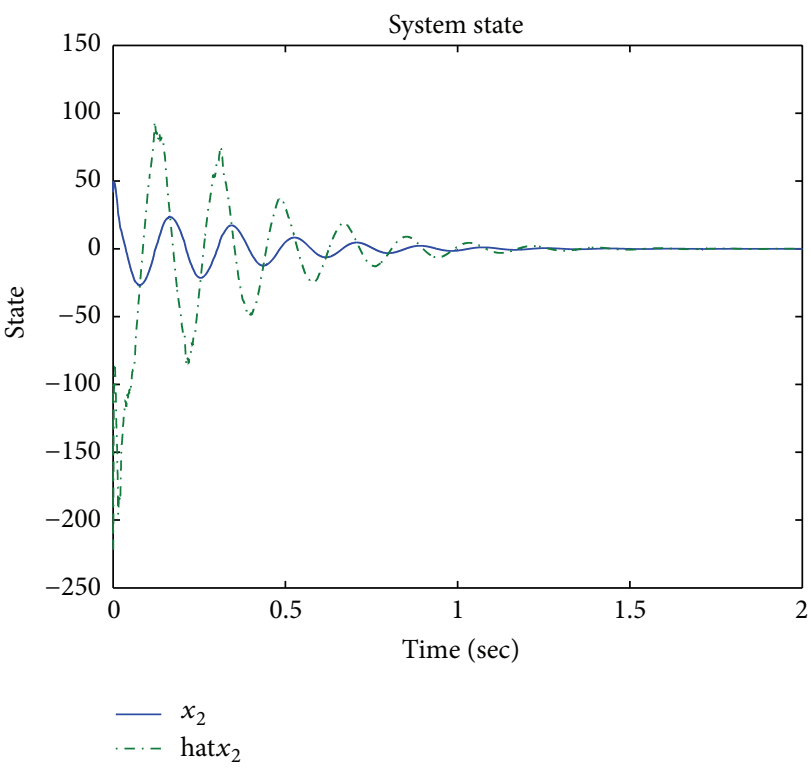

(b)

FIGURE 1: The state response.

\section{Conclusion}

In this paper, we combine the backstepping method with the mathematical induction to prove that a class of stochastic nonlinear systems with time-varying delays by the output feedback control is globally asymptotically stable in probability. It should be pointed out that we not only extend the results in deterministic nonlinear systems to more complex stochastic case but also add time-varying delays to stochastic nonlinear systems. For the term of time-varying delays, we design a Lyapunov-Krasovskii functional and a linear output feedback controller to render the closed-loop system globally asymptotically stable. 


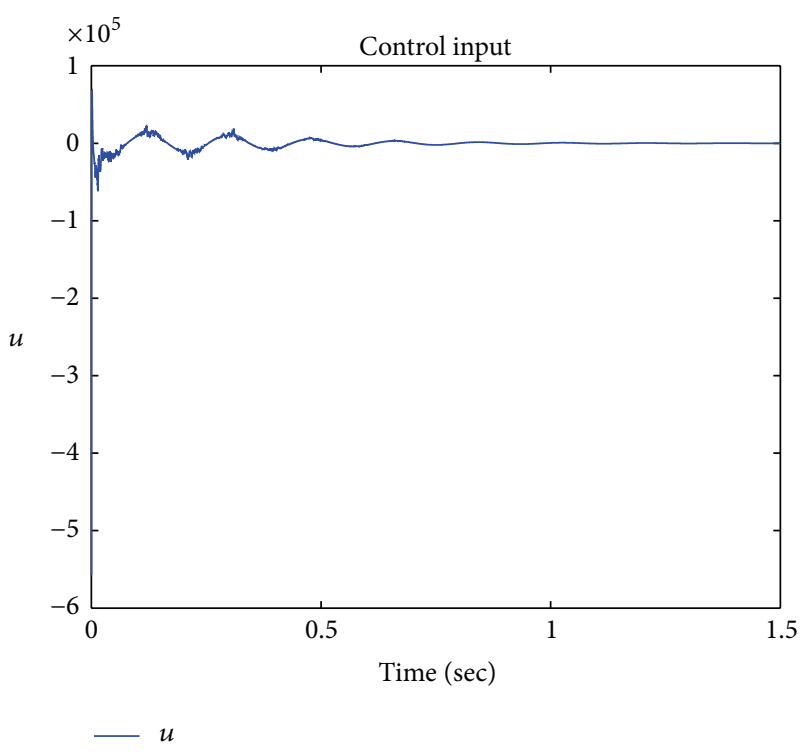

FIgURE 2: The control input.

\section{Conflict of Interests}

The authors declare that there is no conflict of interests regarding the publication of this paper.

\section{Acknowledgments}

This work was jointly supported by the Alexander von Humboldt Foundation of Germany (Fellowship CHN/ 1163390), the National Natural Science Foundation of China (61374080), the Priority Academic Program Development of Jiangsu Higher Education Institutions, and the Play of Nature Science Fundamental Research in Nanjing Xiao Zhuang University (2012NXY12).

\section{References}

[1] I. Y. Kats and A. A. Martynyuk, Stability and Stabilization of Nonlinear Systems with Random Structure, Taylor \& Francis, London, UK, 2002.

[2] R. Marino and P. Tomei, "Nonlinear output feedback tracking with almost disturbance decoupling," IEEE Transactions on Automatic Control, vol. 44, no. 1, pp. 18-28, 1999.

[3] S. Battilotti, "Global output regulation and disturbance attenuation with global stability via measurement feedback for a class of nonlinear systems," IEEE Transactions on Automatic Control, vol. 41, no. 3, pp. 315-327, 1996.

[4] Z. Pan and T. Basar, "Adaptive controller design for tracking and disturbance attenuation in parametric strict-feedback nonlinear systems," IEEE Transactions on Automatic Control, vol. 43, no. 8, pp. 1066-1083, 1998.

[5] Z. Pan and T. Basar, "Backstepping controller design for nonlinear stochastic systems under a risk-sensitive cost criterion," SIAM Journal on Control and Optimization, vol. 37, no. 3, pp. 957-995, 1999.

[6] Z. Pan, Y. Liu, and S. Shi, "Output feedback stabilization for stochastic nonlinear systems in observer canonical form with stable zero-dynamics," Science in China, vol. 44, no. 4, pp. 292308, 2001.

[7] H. Deng and M. Krstić, "Stochastic nonlinear stabilization-I: a backstepping design," Systems \& Control Letters, vol. 32, no. 3, pp. 143-150, 1997.

[8] H. Deng and M. Krstić, "Stochastic nonlinear stabilization-II: inverse optimality," Systems \& Control Letters, vol. 32, no. 3, pp. 151-159, 1997.

[9] H. Deng and M. Krstić, "Output-feedback stochastic nonlinear stabilization," IEEE Transactions on Automatic Control, vol. 44, no. 2, pp. 328-333, 1999.

[10] H. Deng, M. Krstic, and R. J. Williams, "Stabilization of stochastic nonlinear systems driven by noise of unknown covariance," IEEE Transactions on Automatic Control, vol. 46, no. 8, pp. 12371253, 2001.

[11] S.-J. Liu, Z.-P. Jiang, and J.-F. Zhang, “Global output-feedback stabilization for a class of stochastic non-minimum-phase nonlinear systems," Automatica, vol. 44, no. 8, pp. 1944-1957, 2008.

[12] S.-J. Liu and J.-F. Zhang, "Output-feedback control of a class of stochastic nonlinear systems with linearly bounded unmeasurable states," International Journal of Robust and Nonlinear Control, vol. 18, no. 6, pp. 665-687, 2008.

[13] S.-J. Liu, S. S. Ge, and J.-F. Zhang, "Adaptive output-feedback control for a class of uncertain stochastic non-linear systems with time delays," International Journal of Control, vol. 81, no. 8, pp. 1210-1220, 2008.

[14] M.-L. Liu and Y.-G. Liu, "Semi-global stabilization via outputfeedback for a class of uncertain nonlinear systems," Acta Automatica Sinica, vol. 39, no. 12, pp. 2154-2159, 2013.

[15] W. Chen, J. Wu, and L. C. Jiao, "State-feedback stabilization for a class of stochastic time-delay nonlinear systems," International Journal of Robust and Nonlinear Control, vol. 22, no. 17, pp. 19211937, 2012.

[16] H. Du, C. Qian, S. Li, and M. Frye, "Global output feedback stabilization of a class of uppertriangular systems with input delay," in Proceedings of the American Control Conference (ACC '12), pp. 5435-5439, Montréal, Canada, June 2012.

[17] S. Khoo, J. Yin, Z. Man, and X. Yu, "Finite-time stabilization of stochastic nonlinear systems in strict-feedback form," Automatica, vol. 49, no. 5, pp. 1403-1410, 2013.

[18] Z.-J. Wu, X.-J. Xie, and S.-Y. Zhang, "Stochastic adaptive backstepping controller design by introducing dynamic signal and changing supply function," International Journal of Control, vol. 79, no. 12, pp. 1635-1646, 2006.

[19] Z.-J. Wu, X.-J. Xie, and S.-Y. Zhang, "Adaptive backstepping controller design using stochastic small-gain theorem," Automatica, vol. 43, no. 4, pp. 608-620, 2007.

[20] X.-J. Xie and L. Liu, "Further results on output feedback stabilization for stochastic high-order nonlinear systems with time-varying delay," Automatica, vol. 48, no. 10, pp. 2577-2586, 2012.

[21] C. Qian and W. Lin, "Output feedback control of a class of nonlinear systems: a nonseparation principle paradigm," IEEE Transactions on Automatic Control, vol. 47, no. 10, pp. 1710-1715, 2002.

[22] L. Liu and X.-J. Xie, "State feedback stabilization for stochastic feedforward nonlinear systems with time-varying delay," Automatica, vol. 49, no. 4, pp. 936-942, 2013. 
[23] J.-Y. Zhai and W.-T. Zha, "Global adaptive output feedback control for a class of nonlinear time-delay systems," ISA Transactions, vol. 53, no. 1, pp. 2-9, 2014.

[24] N. Duan and X.-J. Xie, "Further results on output-feedback stabilization for a class of stochastic nonlinear systems," IEEE Transactions on Automatic Control, vol. 56, no. 5, pp. 1208-1213, 2011. 


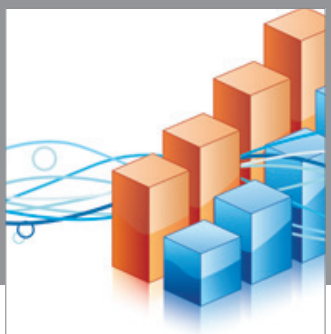

Advances in

Operations Research

vatem alat4

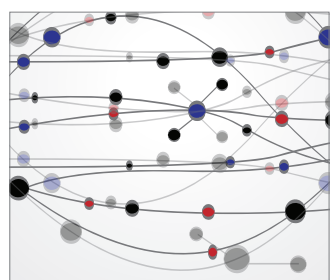

\section{The Scientific} World Journal
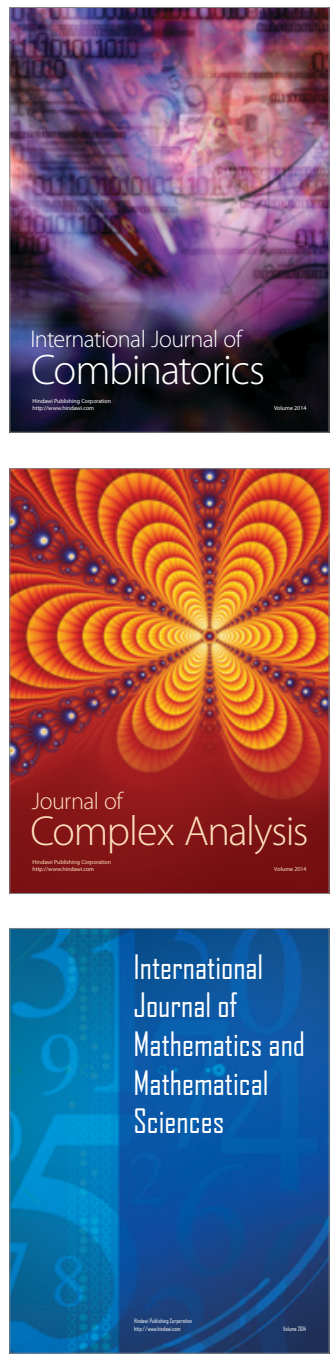
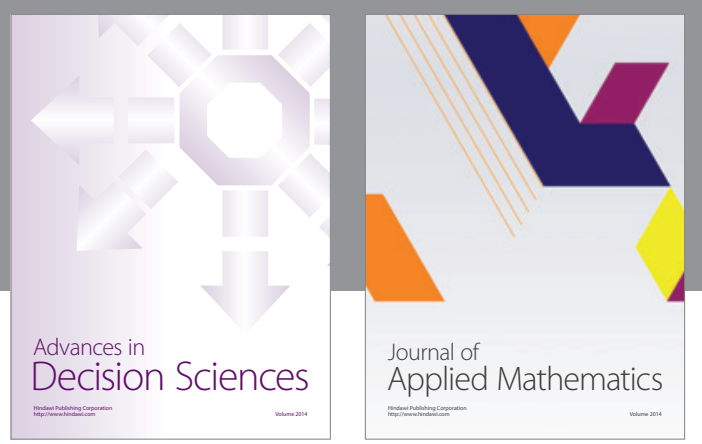

Algebra

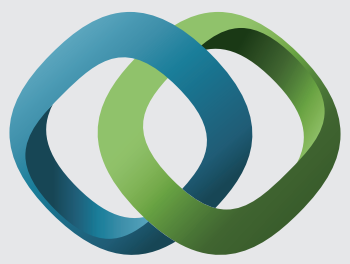

\section{Hindawi}

Submit your manuscripts at

http://www.hindawi.com
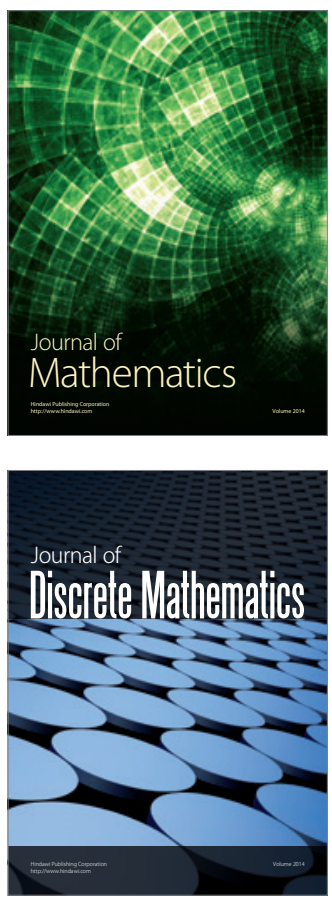

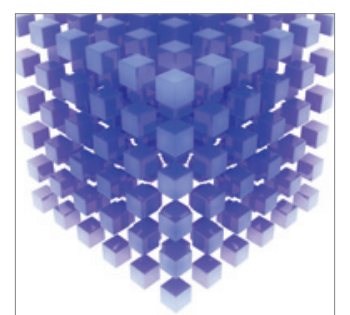

Mathematical Problems in Engineering
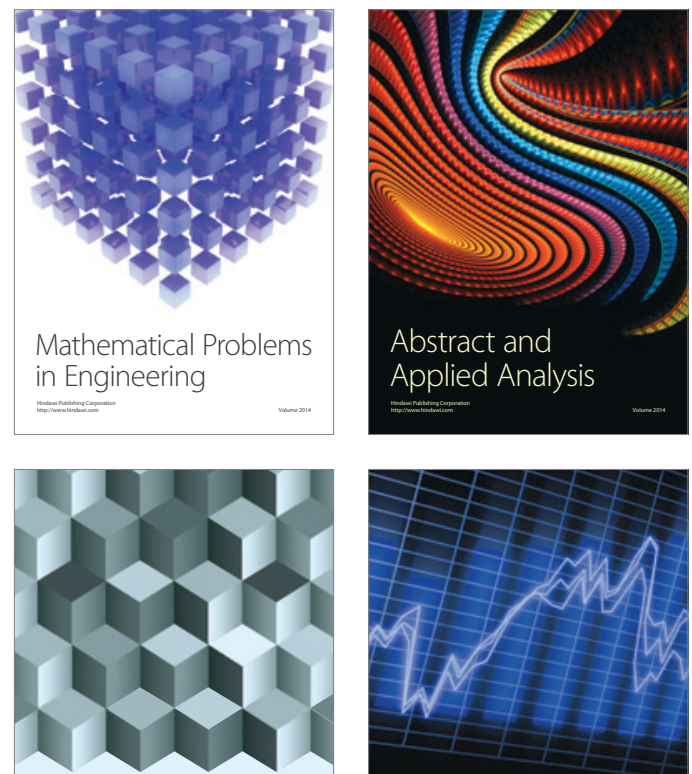

Journal of

Function Spaces

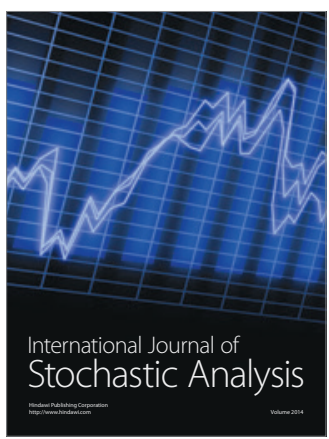

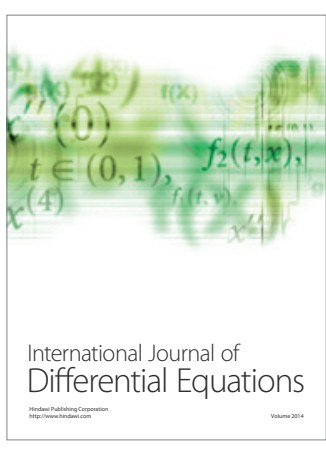
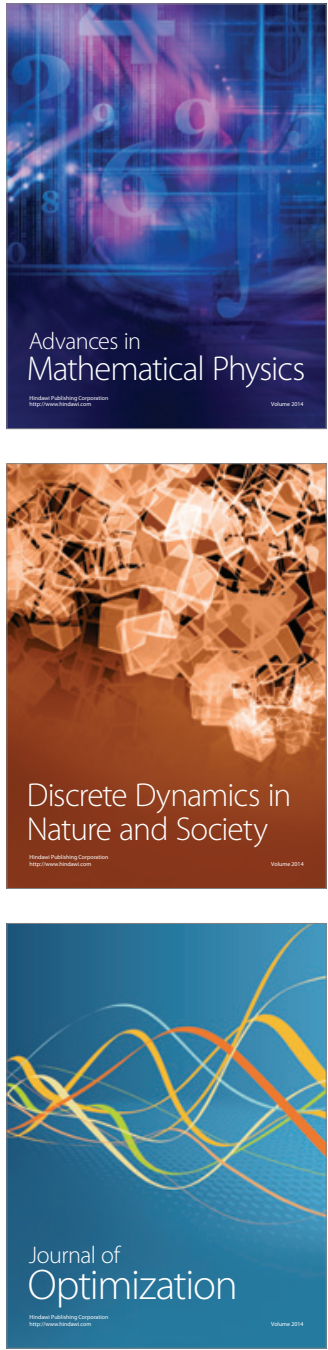